\title{
THE REAL ZEROS OF THE DERIVATIVES OF CYLINDER FUNCTIONS OF NEGATIVE ORDER*
}

\author{
LEE LORCH ${ }^{\dagger}$ AND MARTIN E. MULDOON ${ }^{\ddagger}$
}

To Richard Askey on his 65th birthday

\begin{abstract}
We study the real $x$-zeros of the derivative $C_{\nu}^{\prime}(x)$ of the cylinder function $C_{\nu}(x)=$ $C_{\nu}(x, \alpha)=\cos \alpha J_{\nu}(x)-\sin \alpha Y_{\nu}(x)$ for $\nu<0$. For fixed $\alpha, 0 \leq \alpha<\pi$ and $n=1,2, \ldots$, we show the existence of a number $\nu_{n}$ in the interval $-n+\alpha / \pi-1<\nu<-n+\alpha / \pi$ such that the first positive zero of $C_{\nu}^{\prime}(x)$ occurs after the first positive zero of $C_{\nu}(x)$ when $-n-1+\alpha / \pi<\nu<\nu_{n}$ and before the first positive zero of $C_{\nu}(x)$ when $\nu_{n} \leq \nu<-n+\alpha / \pi$. In case $\nu_{n}<\nu<-n-\alpha / \pi, C_{\nu}^{\prime}(x)$ has two zeros which precede the first positive zero of $C_{\nu}(x, \alpha)$. For $\nu=\nu_{n}, C_{\nu}^{\prime}(-\nu)$ is a double zero. The first is decreasing and the second is increasing as $\nu$ increases. Moreover, in the case $\alpha=0$, for $\nu_{1}=-1.117 \ldots \leq \nu<-1, J_{\nu}^{\prime}(x)$ has exclusively real zeros. The present results and earlier ones on the zeros of $J_{\nu}^{\prime \prime}(x), J_{\nu}^{\prime \prime \prime}(x)$ lead to some conjectures on the zeros of $J_{\nu}^{(n)}(x)$.
\end{abstract}

1. Introduction. The Bessel function $J_{\nu}(x)$ has exclusively real zeros when $\nu \geq-1$, as A. E. Lommel established $[15, \S 15.25$, p. 482] by way of generalizing what J. B. Fourier had shown for $\nu=0$ [ibid.] and the long known results for

$$
J_{-\frac{1}{2}}(x)=\sqrt{\frac{2}{\pi}} \frac{\cos x}{\sqrt{x}}, \quad J_{\frac{1}{2}}(x)=\sqrt{\frac{2}{\pi}} \frac{\sin x}{\sqrt{x}} .
$$

For $-2<\nu<-1$, A. Hurwitz proved ([4], [15, §15.27, pp. 483-485]) that all the zeros of $J_{\nu}(x)$ are real, except for two conjugate purely imaginary zeros, $\pm j_{\nu 1}$. The positive real zeros in this case are $j_{\nu k}, k=2,3, \ldots$. For these $\nu, J_{\nu}(x)$ exhibits other distinctive features, as displayed in the graphs in Figure 1, which shows parts of the graphs of $J_{\nu}(x)$ for the three values $\nu=-1.184,-1.117,-1.05$.

That is, there appears to exist a value $\nu_{1}$ (approximately -1.117) of $\nu$ such that the first maximum of $J_{\nu}(x)$ occurs after the first positive zero when $-2<\nu \leq \nu_{1}$, before the first positive zero when $\nu_{1}<\nu<-1$, with a negative horizontal point of inflection when $\nu=\nu_{1}$.

We shall prove that this value does exist. More generally, we prove a corresponding result for the zeros of $J_{\nu}^{\prime}(x)$ on each of the intervals $(-n-1,-n), n=1,2 \ldots$ and indeed for the zeros of the derivative of the general cylinder function ( $\alpha$ independent of $x$ and $\nu$ )

$$
C_{\nu}(x)=C_{\nu}(x, \alpha)=\cos \alpha J_{\nu}(x)-\sin \alpha Y_{\nu}(x)
$$

on the intervals $-n+\alpha / \pi-1<\nu<-n+\alpha / \pi, n=1,2, \ldots$. In each case we shall show that, as $\nu \uparrow-n+\alpha / \pi$, the abscissa of the first turning point decreases steadily to 0 , while that of the second increases. We shall also establish some inequalities.

In the case $(\alpha=0)$ of the zeros of $J_{\nu}^{\prime}(x)$ in the interval $-2<\nu<-1$ these results parallel some already established for the zeros of $J_{\nu}^{\prime \prime}(x)$ for the interval $-1<\nu<0$

${ }^{*}$ Received April 6, 1998, revised May 29, 1999. Research partially supported by grants from the Natural Sciences and Engineering Research Council (Canada).

†Department of Mathematics and Statistics, York University, Toronto, ON M3J 1P3, Canada (lorch@mathstat.yorku.ca)

$\ddagger$ Department of Mathematics and Statistics, York University, Toronto, ON M3J 1P3, Canada (muldoon@yorku.ca). 


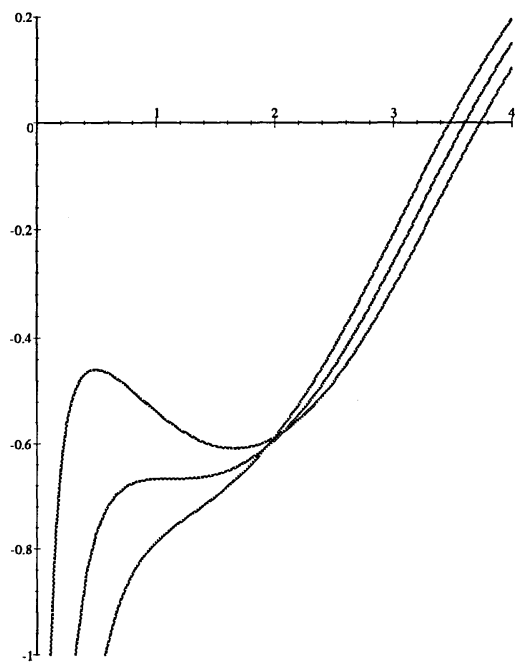

FiguRE $1 . J_{\nu}(x)$ versus $x$, for the values:

(a) $\nu=-1.105$; (b) $\nu=-1.117$; (c) $\nu=-1.184$.

(a) begins closest to the vertical axis.

[13] and for those of $J_{\nu}^{\prime \prime \prime}(x)$ for the interval $0<\nu<1$ [12], thus suggesting that these results, too, can be extended to cylinder functions of appropriate negative order. Our results, together with what is known already, give a complete description of the existence and monotonicity (in $\nu$ ) of real zeros of derivatives of cylinder functions. For the cylinder function $C_{\nu}(x)$ itself, the situation is rather simple: the positive real zeros are all increasing functions of $\nu$ on their intervals of definition. For the derivative $C_{\nu}^{\prime}(x)$, the situation is more complicated as we show here. Many of our results are suggested by the results and numerical investigations of Kerimov and Skorokhodov ([6], [7], [8] and other references.)

2. Notation and main theorem. The formulations of our present result and of the conjectures become more compact if we introduce a notation which enumerates in all cases only the positive (real) zeros. This is because as $\nu$ changes, real zeros may change to non-real and vice versa so the concept of "first" positive zero may give trouble if the standard notations $c_{\nu k}, c_{\nu k}^{\prime}, c_{\nu k}^{\prime \prime}$, etc. are used. We define $c_{\nu k}^{(n)}$ to be the $k$-th positive zero of $C_{\nu}^{(n)}(x)$, with the similar notation $j_{\nu k}^{(n)}$ for the $k$-th positive zero of $J_{\nu}^{(n)}(x)$ and $y_{\nu k}^{(n)}$ for the $k$-th positive zero of $Y_{\nu}^{(n)}(x)$. For $\nu>-1, j_{\nu k}^{(0)}=j_{\nu k}$, the traditional notation. However, for $-2<\nu<-1, j_{\nu k}^{(0)}=j_{\nu, k+1}$, in accordance with Hurwitz's theorem. Similar remarks apply to the zeros $j_{\nu k}^{(n)}$ for $n \geq 1$.

The open interval $(-n-1+\alpha / \pi,-n+\alpha / \pi)$ will be denoted by $I(n, \alpha)$.

We shall prove the following:

THEOREM 2.1. For each $n=1,2, \ldots$, there exists a unique value $\nu_{n}=\nu_{n}(\alpha)$ in 
$I(n, \alpha)$ with the properties that

(i) $c_{\nu 1}^{(1)}=-\nu$ is a double zero of $C_{\nu}^{\prime}(x)$ when $\nu=\nu_{n}$;

(ii) $c_{\nu 1}^{(1)}<-\nu<c_{\nu 2}^{(1)}<c_{\nu 1}^{(0)}<c_{\nu 3}^{(1)}$ when $\nu_{n}<\nu<-n+\alpha / \pi$;

(iii) $c_{\nu 1}^{(1)}>c_{\nu 1}^{(0)}$, when $-n+\alpha / \pi-1<\nu<\nu_{n}$;

(iv) $c_{\nu 1}^{(1)} \downarrow 0$ and $c_{\nu 2}^{(1)} \uparrow c_{\beta 1}^{(1)}, \beta=-n+\alpha / \pi$, as $\nu \uparrow$ in $\nu_{n}<\nu<-n+\alpha / \pi$;

(v) For $k=2,3 \ldots, c_{\nu k}^{(1)}$ is an increasing function of $\nu$ in $I(n, \alpha)$, and $c_{\nu 1}^{(1)}$ is an increasing function of $\nu$ when $-n+\alpha / \pi-1<\nu<\nu_{n}$.

A consequence of these results is that for $\nu<0$, every zero of $C_{\nu}^{\prime}(x)$ which exceeds $-\nu$ is an increasing function of $\nu$ while every zero on $0<x<-\nu$ is a decreasing function of $\nu$.

Remark. For each fixed $n=1,2, \ldots$, it can be shown that $\nu_{n}(\alpha)$ is a decreasing function of $\alpha, 0 \leq \alpha<\pi$.

3. Preliminary results. The Bessel function satisfies the differential equation

$$
x^{2} y^{\prime \prime}+x y^{\prime}+\left(x^{2}-\nu^{2}\right) y=0 .
$$

Every nontrivial solution of (3.1) is a nonzero multiple of (1.1).

When $\nu \in I(n, \alpha), n=1,2, \ldots$, we have $C_{\nu}(0+)= \pm \infty$ and for the discussion of the zeros, we can assume, without loss of generality (replacing the solution by its negative, if necessary) that $C_{\nu}(0+)=-\infty$ while $C_{\nu}^{\prime}(0+)=+\infty$. Hence, if $c_{\nu k}^{(1)}<c_{\nu 1}^{(0)}$, then $C_{\nu}\left(c_{\nu k}^{(1)}\right)<0$. Using this information in (3.1) with $x=c_{\nu 1}^{(1)}$, we note that the first term is non-positive and the second is zero. Hence the third must be non-negative. When $c_{\nu 1}^{(1)}<c_{\nu 1}^{(0)}$ the factor of $\left[c_{\nu 1}^{(1)}\right]^{2}-\nu^{2}$ is negative, so that $\left[c_{\nu 1}^{(1)}\right]^{2} \leq \nu^{2}$ if $c_{\nu 1}^{(1)}<c_{\nu 1}^{(0)}$.

Similarly, $\left[c_{\nu 2}^{(1)}\right]^{2}>\nu^{2}$ if $c_{\nu 1}^{(1)}<c_{\nu 2}^{(1)}<c_{\nu 1}^{(0)}$, since in this case $c_{\nu 2}^{(1)}$ gives rise to a local minimum. Were $c_{\nu 3}^{(1)}$ also to precede $c_{\nu 1}^{(0)}$, we would have the contradiction that $\nu^{2}<\left[c_{\nu 2}^{(1)}\right]^{2}<\left[c_{\nu 3}^{(1)}\right]^{2}<\nu^{2}$. Further, it is clear that if $c_{\nu 1}^{(1)}$, which gives rise to a local maximum or an inflection-point, precedes $c_{\nu 1}^{(0)}$, then $c_{\nu 2}^{(1)}<c_{\nu 1}^{(0)}$ unless $c_{\nu 1}^{(1)}$ yields an inflection-point. In the latter case $\left[c_{\nu 1}^{(1)}\right]^{2}=\nu^{2}$, in the former, $\left[c_{\nu 1}^{(1)}\right]^{2}<\nu^{2}<\left[c_{\nu 2}^{(1)}\right]^{2}$.

Summarizing, we have

THEOREM 3.1. If $\nu \in I(n, \alpha)$, and if $c_{\nu 1}^{(1)}<c_{\nu 1}^{(0)}$, then either $\left[c_{\nu 1}^{(1)}\right]^{2}<\nu^{2}<$ $\left[c_{\nu 2}^{(1)}\right]^{2}<\left[c_{\nu 1}^{(0)}\right]^{2}<\left[c_{\nu 3}^{(1)}\right]^{2}$ or $c_{\nu 1}^{(1)}=-\nu$ is a double zero of $C_{\nu}^{\prime}(x)$.

Now is a good moment to recall a formula due to Watson $[15, \S 15.6(4)$, p. 510], namely

$$
\frac{d c^{\prime}}{d \nu}=\frac{2 c^{\prime}}{c^{2}-\nu^{2}} \int_{0}^{\infty}\left(c^{\prime 2} \cosh 2 t-\nu^{2}\right) K_{0}\left(2 c^{\prime} \sinh t\right) e^{-2 \nu t} d t
$$

where we write $c^{\prime}$ for $c_{\nu k}^{(1)}$. Together with Theorem 3.1, the Watson formula yields immediately the first half of Theorem $2.1(\mathrm{v})$. We state it separately now as part of the next result.

THEOREM 3.2. If $\nu \in I(n, \alpha)$, then $c_{\nu k}^{(1)}$ is an increasing function of $\nu$ for each fixed $k=2,3, \ldots$ Moreover, if $c_{\nu 1}^{(1)}>c_{\nu 1}^{(0)}$, then also $c_{\nu 1}^{(1)}$ is an increasing function of $\nu$ in $I(n, \alpha)$. 
Proof. The first assertion is verified by (3.2) and Theorem 3.1. To justify the seconcl statement, it suffices to establish that in this case $\left[c_{\nu 1}^{(1)}\right]^{2}>\nu^{2}$.

Substituting $x=c_{\nu 1}^{(1)}$ in (3.1), we find that $\left[c_{\nu 1}^{(1)}\right]^{2} \geq \nu^{2}$, since $c_{\nu 1}^{(1)}$ gives now a positive maximum (in which case $\left[c_{\nu 1}^{(1)}\right]^{2}>\nu^{2}$ ) or a positive inflection point (in which case $\left.\left[c_{\nu 1}^{(1)}\right]^{2}=\nu^{2}\right)$.

The latter cannot occur. To see this, replace $x^{2}$ in (3.1) by a value slightly larger than $\nu^{2}$. The third term becomes positive. The middle term would also have to be positive; otherwise $c_{\nu 1}^{(1)}$ would have given a maximum. Thus the first term would be negative. This, however, would require $C_{\nu}^{\prime}(x)$ to decrease from zero after passing $x=-\nu$, again a situation which makes the supposed point of inflection really a maximum. Hence, $C_{\nu}^{\prime}(x)$ cannot have a point of inflection at $c_{\nu 1}^{(1)}$ when $c_{\nu 1}^{(1)}>c_{\nu 1}^{(0)}$. This completes the proof of the theorem. Further, it is clear from the differential equation (3.1), by way of a converse to part of Theorem 3.1, that if $C_{\nu}^{\prime}(-\nu)=0, \nu \neq 0$, then $x=-\nu$ is a double zero of $C_{\nu}^{\prime}(x)$.

In the special case $\alpha=0$, applying the fsolve command of Maple V, Version 4 to either $J_{\nu}^{\prime}(-\nu)=0$ or to $J_{\nu}^{\prime \prime}(-\nu)=0,-2<\nu<-1$, yields the value $\nu=$ $-1.117123077, \ldots$, i.e., the value $\nu_{1}$, the same as calculated otherwise in [2] and [7]. This provides a numerical support for Theorem 2.1 (i).

4. Further results and proof of Main Theorem. In the interval $I(n, \alpha),[15$, $\S 15.6$, pp. 508- 509], each $c_{\nu k}$ varies continuously, including one-sided continuity at the end-points. Moreover, $\left[15, \S 15.6\right.$, p. 509], no zero of $C_{\nu}(x, \alpha)$ either appears or disappears as $\nu$ moves in $I(n, \alpha)$. These properties depend on Watson's formula [15, $\S 15.6$ (3), p. 509] for $d c / d \nu$, which is analytic in $\nu$. We need below, e.g., in the proof of Theorem 4.6, the consequence that $c$ increases with $\nu$ in $I(n, \alpha)$.

For: $C_{\nu}^{\prime}(x)$, the situation is somewhat altered, since the analyticity of $d c^{\prime} / d \nu$, on which we must now rely, is more restricted. Here the relevant Watson formula for $d c^{\prime} / d \nu\left[15, \S 15.6(4)\right.$, p. 510], recorded here as $(3.2)$, has $2 c^{\prime} /\left(c^{\prime 2}-\nu^{2}\right)$ as a factor of the integral. Hence, analyticity of $c^{\prime}$ as a function of $\nu$ cannot be asserted for $c^{\prime 2}=\nu^{2}$. Thus if, as we shall see, there is a unique $\nu_{n}$ such that $C_{\nu_{n}}^{\prime}\left(-\nu_{n}\right)=0$, while the Watson reasoning for $c$ applies to $c^{\prime}$ in the subintervals $\left(-n-1+\alpha / \pi, \nu_{n}\right)$ and $\left(\nu_{n},-n+\alpha / \pi\right)$ of $I(n, \alpha)$, it cannot be used for $I(n, \alpha)$ regarded as a single interval. The function $c^{\prime}$ is continuous in each of these subintervals of $I(n, \alpha)$, including one-sided continuity at the respective end-points of $I(n, \alpha)$.

In other words, while we can infer (à la Watson), that no zero of $C_{\nu}^{\prime}(x)$ can appear or disappear in either $\left(-n-1+\alpha / \pi, \nu_{n}\right)$ or $\left(\nu_{n},-n+\alpha / \pi\right)$, we cannot assert that no new zero can arise in $I(n, \alpha)$. That is, we may have a new zero, say $c^{\prime}$, of $C_{\nu}^{\prime}(x)$ present itself for which $c^{\prime}=-\nu$. Indeed this can happen. From Bessel's differential equation, it is clear that it can happen only when $x^{2}=\nu^{2}$.

LEMMA 4.1. The ratio $J_{\nu}^{\prime}(|\nu|) / Y_{\nu}^{\prime}(|\nu|)$ increases from $-\infty$ to $\infty$ between consecutive zeros of $Y_{\nu}^{\prime}(|\nu|),-\infty<\nu<\infty$; between the first zero $\nu=-0.5971610161$ of $Y_{\nu}^{\prime}(|\nu|)$ and $\nu=0$, the ratio increases from $-\infty$ to 0 .

Proof. For $\nu>0$, where the denominator is never zero, this is already known [14, (2)]. For $\nu<0$, and for the interval between 0 and the first zero of $Y_{\nu}^{\prime}(|\nu|)$, the cases to be applied below, the proof is the same as that for $[14,(2)]$, except that $x$ must be replaced by the positive quantity $-\nu$ in the expression two lines below [14, (2)] instead of by $\nu$. 

$C_{\nu}^{\prime}(x)$.

We require somewhat more. Preliminary to that, we need a further property of

LEMMA 4.2. If $x^{2} \geq \nu^{2}$, the positive zeros of the derivatives $C_{\nu}^{\prime}(x), \bar{C}_{\nu}^{\prime}(x)$ of any pair of linearly independent cylinder functions are interlaced.

Proof. The assumption that $x^{2} \geq \nu^{2}$ implies (via Bessel's differential equation) that each maximum of either function is positive, each minimum negative.

Let, with $\alpha \delta-\beta \gamma>0$,

$$
C_{\nu}(x)=\alpha J_{\nu}(x)+\beta Y_{\nu}(x), \bar{C}_{\nu}(x)=\gamma J_{\nu}(x)+\delta Y_{\nu}(x) .
$$

Then

$$
f(x):=C_{\nu}(x) \bar{C}_{\nu}^{\prime}(x)-\bar{C}_{\nu}(x) C_{\nu}^{\prime}(x)=\frac{2(\alpha \delta-\beta \gamma)}{\pi x}>0
$$

Now, $f\left(c_{\nu k}^{(1)}\right)=C_{\nu}\left(c_{\nu k}^{(1)}\right) \bar{C}_{\nu}^{\prime}\left(c_{\nu k}^{(1)}\right)>0, \quad k=1,2, \ldots$. Inasmuch as the successive $C_{\nu}\left(c_{\nu k}^{(1)}\right)$ alternate in sign, so do the successive $\bar{C}_{\nu}^{\prime}\left(c_{\nu k}^{(1)}\right)$. Hence $\bar{C}_{\nu}^{\prime}(x)$ has an odd number of zeros between a pair of successive zeros of $C_{\nu}^{\prime}(x)$. Considering now $f\left(\bar{c}_{\nu k}^{(1)}\right)$ where $\bar{C}_{\nu}^{\prime}\left(\bar{c}_{\nu k}^{(1)}\right)=0$, we see that $C_{\nu}^{\prime}(x)$ has an odd number of zeros between any pair $\bar{c}_{\nu k}^{(1)}, \bar{c}_{\nu, k+1}^{(1)}$. This establishes the interlacing asserted.

With this property known, we can extend Lemma 4.1.

LEMmA 4.3. The function $J_{\nu}^{\prime}(-\nu) / Y_{\nu}^{\prime}(-\nu), \nu<0, \nu \in I(n, \alpha)$, for a fixed $n=1,2, \ldots$, is univalent and piecewise increasing in $c_{\nu k}^{(1)}<-\nu<c_{\nu, k+1}^{(1)}$.

Proof. We consider $C_{\nu}$ and $Y_{\nu}$ to be linearly independent, the case where they are linearly dependent being covered by Lemma 4.1. The zeros of $C_{\nu}^{\prime}(x)$ and $Y_{\nu}^{\prime}(x), x \geq$ $-\nu$, are interlaced, according to Lemma 4.2. We distinguish two cases: (a) $y_{\nu k}^{(1)}<c_{\nu k}^{(1)}$ and (b) $y_{\nu k}^{(1)}>c_{\nu k}^{(1)}$. In case (a), applying Lemma 4.1, the function $J_{\nu}^{\prime}(-\nu) / Y_{\nu}^{\prime}(-\nu)$ is increasing in $y_{\nu k}^{(1)}<c_{\nu k}^{(1)}<-\nu<y_{\nu, k+1}^{(1)}$ and again in $y_{\nu, k+1}^{(1)}<-\nu<c_{\nu, k+1}^{(1)}<y_{\nu, k+2}^{(1)}$. However,

$$
\frac{J_{\nu}^{\prime}\left(c_{\nu k}^{(1)}\right)}{Y_{\nu}^{\prime}\left(c_{\nu k}^{(1)}\right)}=\tan \alpha=\frac{J_{\nu}^{\prime}\left(c_{\nu, k+1}^{(1)}\right)}{Y_{\nu}^{\prime}\left(c_{\nu, k+1}^{(1)}\right)}
$$

and so the Lemma holds in this case.

Case (b) can be included in case (a), with an obvious change in notation, except for $c_{\nu 1}^{(1)}<y_{\nu 1}^{(1)}$. Here too, Lemma 4.1 shows that $J_{\nu}^{\prime}(-\nu) / Y_{\nu}^{\prime}(-\nu), \quad \nu<0$, increases in $(0<) c_{\nu 1}^{(1)}<-\nu<y_{\nu 1}^{(1)}$ and again in $y_{\nu 1}^{(1)}<-\nu<c_{\nu 2}^{(1)}\left(<y_{\nu 2}^{(1)}\right)$. As before,

$$
\frac{J_{\nu}^{\prime}\left(c_{\nu 1}^{(1)}\right)}{Y_{\nu}^{\prime}\left(c_{\nu 1}^{(1)}\right)}=\frac{J_{\nu}^{\prime}\left(c_{\nu 2}^{(1)}\right)}{Y_{\nu}^{\prime}\left(c_{\nu 2}^{(1)}\right)}=\tan \alpha
$$

so that $J_{\nu}^{\prime}(-\nu) / Y_{\nu}^{\prime}(-\nu)$ is univalent in $c_{\nu 1}^{(1)}<-\nu<c_{\nu 2}^{(1)}$, as asserted.

In sum,

THEOREM 4.4. For a given cylinder function $C_{\nu}(x, \alpha)$, with $\nu<0$, there is exactly one value $\nu_{n}$ of $\nu$ in each $I(n, \alpha)$ for which $C_{\nu}^{\prime}(x)$ has a double zero for $x=-\nu$. 
Proof. Such a value of $\nu$ occurs for $\tan \alpha=J_{\nu}^{\prime}(-\nu) / Y_{\nu}^{\prime}(-\nu)$ and so the existence follows from the increase of $J_{\nu}^{\prime}(-\nu) / Y_{\nu}^{\prime}(-\nu)$ in $I(n, \alpha)$. For the uniqueness, suppose that $\nu=-r$ and $\nu=-s$ each give rise to a double zero in $I(n, \alpha), r<s$. Then $C_{\nu}^{\prime}(r):=C_{\nu}^{\prime}(s)=0$, so that

$$
\frac{J_{\nu}^{\prime}(r)}{Y_{\nu}^{\prime}(r)}=\frac{J_{\nu}^{\prime}(s)}{Y_{\nu}^{\prime}(s)}=\tan \alpha .
$$

But this is impossible in view of the univalence assertion in Lemma 4.3.

Next we show that increasing $\nu$ past $\nu_{n}$ in $I(n, \alpha)$ causes the double zero to split into a pair of simple zeros, each still smaller than $c_{\nu 1}$.

THEOREM 4.5. If $C_{\lambda}^{\prime}(x)$ has a double zero, $x=-\lambda$, in $I(n, \alpha)$ for some fixed $n=1,2, \ldots$, and if $0>\mu>\lambda, \mu \in I(n, \alpha)$, then $\left[c_{\mu 1}^{(1)}\right]^{2}<\mu^{2}<\left[c_{\mu 2}^{(1)}\right]^{2}<\left[c_{\mu 1}^{(0)}\right]^{2}$.

Proof. We consider the case where $C_{\lambda}(0)=-\infty$. When $C_{\lambda}(0)=+\infty$, only minor changes in the proof will be required. Here, therefore, $C_{\lambda}(-\lambda)<0$.

Motivated by Bessel's differential equation in the form

$$
\left(x y^{\prime}\right)^{\prime}+\frac{x^{2}-\nu^{2}}{x} y=0, \quad y=C_{\nu}(x)
$$

we define $W(x)=\left(x y^{\prime}\right) Y-\left(x Y^{\prime}\right) y$, where now $y=C_{\lambda}(x)$ and $Y=C_{\mu}(x)$. Then

$$
x W^{\prime}=\left(\lambda^{2}-\mu^{2}\right) y Y .
$$

Therefore, $W^{\prime}(x)>0$ and so $W(x) \uparrow$ when $0<x<c_{\lambda 1}^{(0)}$, since $c_{\mu 1}^{(0)}>c_{\lambda 1}^{(0)}$, while $C_{\lambda}(0)=-\infty$. Since $W\left(c_{\lambda 1}^{(0)}\right)=c_{\lambda 1}^{(0)} C_{\lambda}^{\prime}\left(c_{\lambda 1}^{(0)}\right) C_{\mu}\left(c_{\lambda 1}^{(0)}\right)<0$, we have

$$
0>W(-\lambda)=-\lambda C_{\lambda}^{\prime}(-\lambda) C_{\mu}(-\lambda)-(-\lambda) C_{\mu}^{\prime}(-\lambda) C_{\lambda}(-\lambda)=\lambda C_{\mu}^{\prime}(-\lambda) C_{\lambda}(-\lambda)
$$

and so $C_{\mu}^{\prime}(-\lambda)<0$.

Thus $C_{\mu}(x)$, which is $-\infty$ when $x=0$, has passed a local maximum by the time $x=-\lambda$. Accordingly, $c_{\mu 1}^{(1)}<-\lambda<c_{\lambda 1}^{(0)}<c_{\mu 1}^{(0)}$, so that $C_{\mu}\left(c_{\mu 1}^{(1)}\right)<0$. Hence, $c_{\mu 1}^{(1)}<c_{\mu 2}^{(1)}<c_{\mu 1}^{(0)}$, since $C_{\mu}(x)$ must have a local minimum before it reaches 0 at $x=c_{\mu 1}^{(0)}$.

As part of the proof of Theorem 2.1, we need to verify its assertion (iv). For convenience, we restate this as a separate Theorem.

THEOREM 4.6. If $\nu<0$ and if $c_{\nu 1}^{(1)}<c_{\nu 1}^{(0)}$, then (i) $c_{\nu 1}^{(1)} \downarrow 0$ and (ii) $c_{\nu 2}^{(1)} \uparrow$ $c_{-n+\alpha / \tau, 1}^{(1)}$, as $\nu \uparrow-n+\alpha / \pi, n=1,2, \ldots$

Proof. As before, it suffices to consider the case in which $C_{\nu}(0+)=-\infty$. Again, we employ the function $W(x)=\left(x y^{\prime}\right) Y-\left(x Y^{\prime}\right) y$, with $y=C_{\nu}(x), Y=C_{\mu}(x)$, $0>\mu>\nu$, and $\mu, \nu$ both in $I(n, \alpha)$.

Further, $x W^{\prime}=\left(\nu^{2}-\mu^{2}\right) y Y>0, \quad 0<x<c_{\nu 1}^{(0)}$ so that $W(x)$ increases in $0<x<c_{\nu 1}^{(0)}$.

Now,

$$
W\left(c_{\nu 1}^{(0)}\right)=c_{\nu 1}^{(0)} C_{\nu}^{\prime}\left(c_{\nu 1}^{(0)}\right) C_{\mu}\left(c_{\nu 1}^{(0)}\right)
$$


The first two factors are positive. The third is negative, since $c_{\mu 1}^{(0)}>c_{\nu 1}^{(0)}$. Hence $W(x)<0,0<x<c_{\nu 1}^{(0)}$.

Therefore,

$$
0>W\left(c_{\nu k}^{(1)}\right)=-c_{\nu k}^{(1)} C_{\mu}^{\prime}\left(c_{\nu k}^{(1)}\right) C_{\nu}\left(c_{\nu k}^{(1)}\right)
$$

and so $C_{\mu}^{\prime}\left(c_{\nu k}^{(1)}\right)<0, k=1,2$. Thus $c_{\nu 1}^{(1)}>c_{\mu 1}^{(1)}$ and $c_{\mu 2}^{(1)}>c_{\nu 1}^{(1)}$.

This confirms the monotonicity of $c_{\nu k}^{(1)}, k=1,2$, in the direction stated in (i) and (ii), $\nu \in I(n, \alpha)$.

Their respective limits as $\nu \uparrow-n+\alpha / \pi$ remain to be determined. First we note that $\Lambda_{1}=\lim c_{\nu 1}^{(1)} \geq 0$. If $\Lambda_{1}>0$, then by the inequalities proved in Theorem 4.6, there would have to be a $\mu$ with $\lambda<\mu \leq-n+\alpha / \pi$ with the property that $c_{\mu 1}^{(1)}=-\mu$, contradicting the uniqueness assertion of Theorem 4.4. Hence $\Lambda_{1}=0$, as claimed. The limit of $c_{\nu 2}^{(1)}$, an increasing function, follows from the continuity of $c_{\nu 2}^{(1)}$ in $I(n, \alpha)$, especially the one-sided continuity at the (upper) end-point $-n+\alpha / \pi$ of the interval.

The proof of Theorem 4.6 is complete.

We may now complete the proof of Theorem 2.1. The existence of $\nu_{n}(\alpha)$ with property (i) is a consequence of Theorem 4.4. (ii) is a consequence of Theorems 3.1 and 4.5. Part (iii) of Theorem 2.1 follows from Theorem 4.6. If we had $c_{\nu 1}^{(1)}<c_{\nu 1}^{(0)}$, when $-n+\alpha / \pi-1<\nu<\nu_{n}$, then Theorem 4.6 would assert the existence of a pair of zeros with the same monotonicity and the same limits as those arising in part (ii) of the Theorem. This would lead to the existence of double zeros at these limits and would contradict the fact that such double zeros can occur only for $x=-\nu$. Finally, part (iv) of Theorem 2.1 follows from Theorem 4.6, while part (v) follows from part (iii) and Theorem 3.2.

Remark. The increasing character of $c_{\nu k}^{(1)}, k=2,3, \ldots$ in $I(n, \alpha)$ and of $c_{\nu 1}^{(1)}$ in $\left(-n+\alpha / \pi-1, \nu_{n}\right)$ follows from Watson's formula for $d c^{\prime} / d \nu$ since $\left[c_{\nu k}^{(1)}\right]^{2}>\nu^{2}$ in these cases.

CoROllary 4.7. If $x>0$ then

$$
f(x)=\int_{0}^{\infty}\left(x^{2} \cosh 2 t-\nu^{2}\right) K_{0}(2 x \sinh t) e^{-2 \nu t} d t>0 .
$$

Proof. For $x^{2} \geq \nu^{2}$, this is obvious. When $x^{2}<\nu^{2}$, construct the cylinder function $C_{\nu}(x), \nu<0$ for which the specified $x=c_{\nu 1}^{(1)}$. According to Theorem 2.1 (iii), and Watson's formula (3.2)

$$
0>\frac{d x}{d \nu}=\frac{2 x}{x^{2}-\nu^{2}} f(x), x>0, x^{2}<\nu^{2},
$$

and so $f(x)>0$ also when $x^{2}<\nu^{2}$.

Special cases of Theorem 4.4 are among results already found by other methods. Cf. [7] and [8] and the references provided there. In particular, $C_{\nu}(x, 0)=J_{\nu}(x)$ and $C_{\nu}(x, \pi / 2)=Y_{\nu}(x)$. In these respective cases, Theorem 4.4 yields the following Corollaries, already known ([7] and [8]).

Corollary 4.8. On each interval $-n-1<\nu<-n, n=1,2, \ldots$, there is a single value $\nu_{n}$ of $\nu$ for which $J_{\nu}^{\prime}(x)$ has a positive double zero. 
Corollary 4.9. On each interval $-m-1 / 2<\nu<-m+1 / 2, m=1,2, \ldots$, there is a single value $\nu_{m}$ of $\nu$ for which $Y_{\nu}^{\prime}(x)$ has a positive double zero.

5. Reality of zeros. It is well known ([7], [9]) that the smallest positive zero of $J_{\nu}^{\prime}(z)$ approaches 0 as $\nu \downarrow 0$ and that it becomes purely imaginary when $-1<\nu<0$ returning to the origin when $\nu \downarrow-1$. But it becomes real again as $\nu$ is further decreased:

THEOREM 5.1. For $\nu_{1}<\nu<-1$, the zeros of $J_{\nu}^{\prime}(z)$ are all real.

Remark. This adds the interval $\left[\nu_{1},-1\right)$ to the known sets $[0, \infty)$ and $\{-1,-2, \ldots\}$ of real values of $\nu$ for which $J_{\nu}^{\prime}(z)$ has exclusively real zeros. of

Proof of Theorem 5.1. The squares of the non-zero zeros of $J_{\nu}^{\prime}(z)$ are the zeros

$$
h(\nu, z)=\Gamma(\nu+2) \nu^{-1} 2^{\nu} z^{(1-\nu) / 2} J_{\nu}^{\prime}\left(z^{1 / 2}\right)=\nu+1-\frac{(\nu+2) z}{4 \nu}+\frac{(\nu+4) z^{2}}{32 \nu(\nu+2)}-\ldots
$$

Suppose that $h(\nu, z)$ has a nonreal zero for some $\nu$ in the interval in question. Then it has a pair of conjugate complex zeros, which vary continuously with $\nu$. But as $\nu$ increases towards -1 such zeros must become real and at the point where they become real there occurs a double zero of $J_{\nu}^{\prime}\left(z^{\frac{1}{2}}\right)$. But [2], for $-2 \leq \nu \leq-1$, the only such double zero can occur for $\nu=\nu_{1}$. Hence there can be only real zeros of $h(\nu, z)$ for the $\nu$-values in question. Furthermore the series expansion for $h(\nu, z)$ shows that when $\nu$ is close to and less than -1 the smallest real zero of $h(\nu, z)$ is, in fact, positive. Thus all its zeros are positive and so all the zeros of $J_{\nu}^{\prime}(z)$ are positive.

Figure 2 gives a graph of the zero of $h(\nu, z)$ which is in question here.

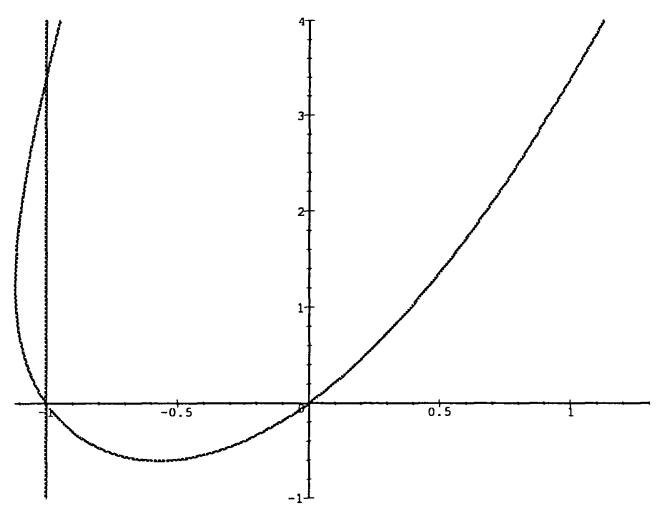

FIGURE 2. Squares of the small zeros of $J_{\nu}^{\prime}(x)$ as functions of $\nu$ (horizontal axis).

6. Cionjectures. Results analogous to what we have found for the special case $c_{\nu k}=j_{\nu 1}$, were found for $j_{\nu k}^{(2)}$ in Theorems 1 and 2 of [13], and for $j_{\nu k}^{(3)}$ in [12]. Together, these three sets of properties suggest the following conjecture:

CONJECTURE 6.1. There exists a unique value $\nu^{(n)}, n-3<\nu^{(n)}<n-2$, such that for $n=1,2, \ldots$, 
(i) $j_{\nu 1}^{(n)}<j_{\nu 2}^{(n)}<j_{\nu 1}^{(0)}<j_{\nu 3}^{(n)}, \nu^{(n)}<\nu<n-2$;

(ii) $j_{\nu 1}^{(n)}>j_{\nu 1}^{(0)}, n-3<\nu<\nu^{(n)}$

(iii) $j_{\nu 1}^{(n)}$ is a double zero of $J_{\nu}^{(n)}(x)$ when $\nu=\nu^{(n)}$;

(iv) $j_{\nu 1}^{(n)} \downarrow 0$ and $j_{\nu 2}^{(n)} \uparrow j_{n-2,1}^{(n)}$ as $\nu \uparrow n-2$;

(v) $j_{\nu k}^{(n)}$ is an increasing function of $\nu, k=2,3, \ldots$, in $n-3<\nu<n-2$, and $j_{\nu 1}^{(n)}$ is an increasing function of $\nu$ in $n-3<\nu<\nu^{(n)}$.

Numerical tests suggest strongly not only the existence of the successive $\nu^{(n)}$ but also simple relations among them, roughly, that the fractional parts decrease and may even be completely monotonic or at least multiply monotonic. This leads to a second conjecture about the real double zeros of $J_{\nu}^{(n)}(x)$ :

Conjecture 6.2. Let $\mu_{n}:=\nu^{(n)}-(n-3)$. Then

$$
\mu_{1}>\mu_{2}>\cdots>\mu_{n}>\mu_{n+1}>\cdots>0
$$

and $\mu_{n}-\mu_{n+1} \downarrow 0$, as $n \rightarrow \infty$. Moreover, the sequence $\left\{\mu_{n}\right\}$ is multiply monotonic, perhaps even completely monotonic.

We display supporting numerical evidence as a table. All available (backward) differences are positive.

\begin{tabular}{|c|c|c|}
\hline$n$ & $\mu_{n}$ & $\mu_{n}-\mu_{n+1}$ \\
\hline \hline 1 & 0.882876923 & 0.082247704 \\
\hline 2 & 0.800629219 & 0.045050926 \\
\hline 3 & 0.7555783929 & 0.027464629 \\
\hline 4 & 0.728113763 & 0.018306585 \\
\hline 5 & 0.709807178 & 0.013021454 \\
\hline 6 & 0.696785724 & 0.009717458 \\
\hline 7 & 0.687068266 & 0.007521549 \\
\hline 8 & 0.679546717 & \\
\hline
\end{tabular}

Our conjecture does not extend to the case $n=0 . J_{\nu}(x)$ has no double zeros except $x=0$ and then only for $\nu>1$ or $\nu=-2,-3, \ldots$.

The decrease of these fractional parts contrasts with (but does not contradict) the increase of the fractional parts noticeable in the table of zeros of $J_{-\nu}^{\prime}(z)$ due to B. Döring [2]. He shows that each interval $k<\nu<k+1, k=1,2, \ldots$, contains precisely one positive double zero of $J_{-\nu}^{\prime}(z)$, as had been conjectured by J. Lense [10]. His table lists those double zeros for $k=1, \ldots, 100$. An examination of this table suggests that the first differences of the fractional parts form a completely monotonic sequence.

M. K. Kerimov and S. L. Skorokhodov ([6], [7], [8]) have investigated, chiefly from the numerical standpoint, double zeros of $J_{\nu}^{(n)}(x)$ for $n=1,2,3$. Values obtained here (using Maple V, Version 4) are consistent with their values which resulted from different software, and also with Döring's.

The regularity of higher differences observed in Döring's table persists, though in a more complicated way, in connection with double zeros of $J_{\nu}^{\prime \prime}(x)$. Here the situation is that there appear to be two values of $\nu$ in each interval $-k-1<\nu<-k, k=1,2, \ldots$ for which double zeros occur. These values occur in two families on the respective 
curves

$$
x=\sqrt{\frac{2 \nu^{2}+1 \pm \sqrt{8 \nu^{2}+1}}{2}} .
$$

The double zeros correspond to roots of

$$
J_{-\nu}^{\prime \prime}\left[\sqrt{\frac{2 \nu^{2}+1 \pm \sqrt{8 \nu^{2}+1}}{2}}\right] .
$$

What appears to be true is that for the upper $(+)$ sign the first differences of the fractional parts of the zeros form a completely monotonic sequence but for the lower $(-)$ sign the second differences of the fractional parts of the zeros form a completely monotonic sequence.

Acknowledgment. We thank a referee for suggestions leading to substantial improvements over the version originally submitted.

\section{REFERENCES}

[1] M. Abramowitz AND I. A. Stegun, Handbook of Mathematical Functions, with Formulas, Craphs and Mathematical Tables, National Bureau of Standards, Applied Mathematics Series 55, Washington, 1964.

[2] B. DöRING, Über die Doppelnullstellen der Ableitung der Besselfunktion, Angew. Informatik, 13 (.l971), pp. 402-406.

[3] A. EFDÉLYI, ET AL, Higher Transcendental Functions, 2, McGraw-Hill, 1953.

[4] A. HuRwitz, Über die Nullstellen der Bessel'schen Function, Math. Ann., 33 (1889), pp. 246-266 (Mathematische Werke, 1, pp. 266-286).

[5] M. E. H. IsmaIl AND M. E. MULDoon, Bounds for the small real and purely imaginary zeros of Bessel and related functions, Methods Appl. Anal., 2 (1995), pp. 1-21.

[6] M. K. KeRIMOV AND S. L. SKoroKHodov, Evaluation of complex zeros of Bessel functions $J_{\nu}(z)$ and $I_{\nu}(z)$ and their derivatives, U. S. S. R. Comput. Math. and Math. Phys., 24:5 (1984), p). 131-141.

[7] M. K. Kerimov And S. L. Skorokhodov, Calculation of the multiple zeros of the derivatives of cylindrical Bessel functions $J_{\nu}(z)$ and $Y_{\nu}(z)$, U. S. S. R. Comput. Math. and Math. Phys., 25i:6 (1985), pp. 101-107.

[8] M. K. KeRIMOV AND S. L. SKoroKhodov, On multiple zeros of derivatives of Bessel's cylindrical functions, Soviet Math. Dokl., 33 (1986), pp. 651-653.

[9] C.G. Kokologiannaki, M. E. Muldoon, and P. D. Siafarikas, A unimodal property of purely inaginary zeros of Bessel and related functions, Canad. Math. Bull., 37 (1994), 365-373.

[10] J. LENSE, Über die Nullstellen der Besselfunktionen und ihrer erster Ableitungen, Jber. Deutschen Math. Verein., 43 (1933/34), pp. 146-153.

[11] L. LoRCH, Monotonicity in terms of order of the zeros of the derivatives of Bessel functions, Proc. Amer. Math. Soc., 108 (1990), 387-389.

[12] L. LoRCH, The zeros of the third derivative of Bessel functions of order less than one, Methods Appl. Anal., 2 (1995), pp. 147-159.

[13] L. Lorch, M. E. Muldoon, AND P. Szego, Inflection points of Bessel functions of negative order, Canad. J. Math., 43 (1991), pp. 1309-1322.

[14] M. E. MUldoon AND R. SPIGLER, Some remarks on zeros of cylinder functions, SIAM J. Math. Anal., 15 (1984), pp. 1231-1233.

[15] G. N. Watson, A Treatise on the Theory of Bessel Functions, 2nd ed., Cambridge University Press, 1944. 